\title{
Azathioprine induced Epstein-Barr virus positive mucocutaneous ulcer: A case report
}

\author{
Sarabjeet Arneja ${ }^{1, *}$, Balsara KP² and Sushil Modhkharkar ${ }^{1}$ \\ ${ }^{1}$ Department of Surgical Pathology and Cytology, Breach Candy Hospital Trust, 60A-Bhulabhai Desai Road, Mumbai- 400026, India \\ 2 Department of GI \& Laparoscopic Colorectal Surgery, Breach Candy Hospital Trust, 60A-Bhulabhai Desai Road, Mumbai- 400026, India
}

\begin{abstract}
Introduction: Epstein-Barr virus positive mucocutaneous ulcer (EBVMUC) is a rare, newly described provisional entity in the 2016 Update of World Health Organization classification of lymphoid neoplasms. The histomorphological and immunophenotypical and molecular features overlap with classical Hodgkin's lymphoma (cHL) and can be mistaken for the same. Case report: A 70-year-old male, a known case of diabetes mellitus and hypertension, was diagnosed with Wegener's granulomatosis (granulomatosis with polyangiitis) in 2007. He was treated with prednisolone and cyclophosphamide, the latter drug was replaced with azathioprine in 2010. He was apparently well since then, until he presented in 2016 with an anal ulcer with a fistula tract formation, the ulcer on histomorphology and immunohistochemistry was diagnosed as EBVMUC. Discussion: EBVMUC was first described in patients with iatrogenic induced immunosuppression. They have later been found to be associated with various other causes of immunosuppression, like solid organ transplant recipients and human immunodeficiency virus (HIV), common factor in all these being immunosuppression. Conclusion: The importance of recognizing this entity lies in its morphological and immunophenotypic overlap with classical Hodgkin's lymphoma (cHL) and unlike latter, most often complete resolution of disease occurs with reduction of immunosuppressive dose. Therefore, correct recognition of the entity is essential to avoid overtreatment as lymphoma.
\end{abstract}

Keywords: Epstein-Barr virus; mucocutaneous ulcer; azathioprine; Wegener's granulomatosis; azathioprine

\section{Introduction}

Epstein-Barr virus positive mucocutaneous ulcer (EBVMUC) is a rare, newly described provisional entity in the 2016 Update of World Health Organization classification of lymphoid neoplasms [1]. It is a localized Epstein-Barr virus (EBV) associated lymphoproliferative disorder, first described as an entity by Dojcinov et al. [2]. The initial series of cases included elderly immunocompetent patients receiving therapeutic immunosuppression. The lesion is a circumscribed shallow ulcer involving oropharyngeal mucosa, skin, or gastrointestinal tract. Irrespective of site of lesion, no associated mass lesion is detectable on imaging or clinical examination [3]. Biopsy specimens revealed a characteristichistology,comprisingapolymorphousreactive infiltrate admixed with large, atypical cells, morphologically and immunophenotypically indistinguishable from Hodgkin/Reed-Sternberg (HRS) cells. The large B-cells are positive for EBV by in situ hybridization and also by immunohistochemistry [2]. The histomorphological and immunophenotypical and molecular features overlap with classical Hodgkin's lymphoma $(\mathrm{cHL})$ and can be mistaken for the same. However, $\mathrm{CHL}$ if left untreated has a five year survival rate of less than $5 \%$ [4]. Unlike $\mathrm{CHL}$ most EBVMUC cases show indolent course, reduction in immunosuppression appeared was sufficient for those receiving immunosuppression. Most cases do not have a relapse of the disease, although occasional cases showed a relapsing and remitting course, but without disease progression [2]. Herein, we report a case of EBVMUC who *Corresponding author: Dr Sarabjeet Arneja, Head-Department of
Surgical Pathology and Cytology, Breach Candy Hospital Trust, 60A-
Bhulabhai Desai Road, Mumbai- 400026, India. Email: drsarabjeetkaur@
breachcandyhospital.org
Received 11 October 2017 Revised 16 January 2018 Accepted 23 January 2018 Published 30 January 2018

Citation: Arneja S, Balsara KP, Modhkharkar S. Azathioprine induced Epstein-Barr virus positive mucocutaneous ulcer: A case report. J Mod Hum Pathol. 2018; 3(1):1-3. DOI: 10.14312/2397-6845.2018-1

Copyright: (c) 2018 Arneja S, et al. Published by NobleResearch Publishers. This is an open-access article distributed under the terms of the Creative Commons Attribution License, which permits unrestricted use, distribution and reproduction in any medium, provided the original author and source are credited. 
had a history of Wegener's granulomatosis (granulomatosis with polyangiitis) treated with azathioprine.

\section{Case report}

A 70-year-old male, non-smoker and non-drinker, known case of diabetes mellitus and hypertension, was diagnosed with Wegener's granulomatosis (granulomatosis with polyangiitis) in 2007. He had presented then with intractable nosebleed, recurrent sinusitis, anterior uveitis, severe joint pain and laboratory findings of rheumatoid (Rh) factor of $45 \mathrm{lU} / \mathrm{ml}$ (normal $15 \mathrm{lU} / \mathrm{ml}$ ) and c-ANCA (cytoplasmic antineutrophil cytoplasmic antibody) of greater than $200 \mathrm{U} / \mathrm{ml}$ (normal $<3 \mathrm{U} / \mathrm{ml}$ ). Since then he was on prednisolone $5 \mathrm{mg}$ and cyclophosphamide. The latter drug was replaced by azathioprine 150 mg daily in 2010 as the patient developed leucopenia and skin darkening. He was apparently well since then, until he presented at our hospital in 2016 with history of pain in the anal region while passing stools and occasional per rectal bleed. Examination under anesthesia (EUA) revealed an anal ulcer 2 - $3 \mathrm{~cm}$ from anal verge. The patient was started on a course of antibiotics and anti-amoebic treatment, but the ulcer did not respond. He presented again, after five months with persistent symptoms this time also associated with purulent discharge. EUA now revealed an anal ulcer with a fistula tract formation (Figure 1).
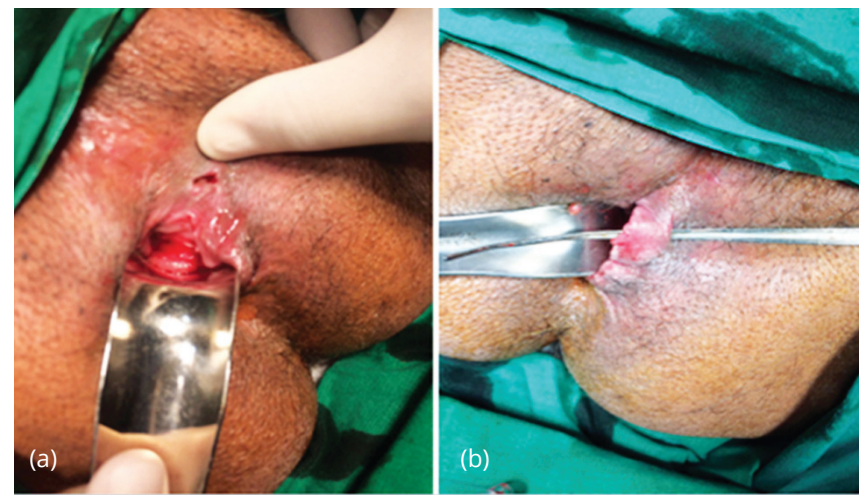

Figure 1a,b Anal ulcer with fistula tract formation.

The fistula tract was laid open and biopsy was taken from the edges of the ulcer and sent for histopathological examination. Microscopy revealed a mucosal ulcer, with underlying dense mixed inflammatory infiltrate within the submucosa composed predominantly of lymphocytes, histiocytes and neutrophils admixed with large atypical, mononuclear to some binuclear cells, with prominent nucleoli, reminiscent of Hodgkin/Reed-Sternberg (HRS) cells seen in $\mathrm{CHL}$ (Figure 2). On immunohistochemistry (Figures 3 \& 4) these large pleomorphic cells expressed an immunophenotype similar to HRS cells of $\mathrm{cHL}$, the cells were immunopositive for CD30, CD15 (variable), PAX5, CD20 and EBV- LMP1 (Epstein-Barr virus-encoded latent membrane protein 1). Cells were immunonegative for leukocyte common antigen(LCA) and CD68. The background lymphocytes showed an admixture of B and T-lymphocytes, highlighted by CD20 and CD3, respectively. The patient was investigated for presence of lymphadenopathy, whole body PET-CT did not show any other mass lesion in the body, in the lymphoid organ or otherwise so the lesion was labelled as EBV induced mucocutaneous ulcer. Following this patient's dose of azathioprine was reduced to $100 \mathrm{mg}$ a day and we are waiting a six-month follow-up.

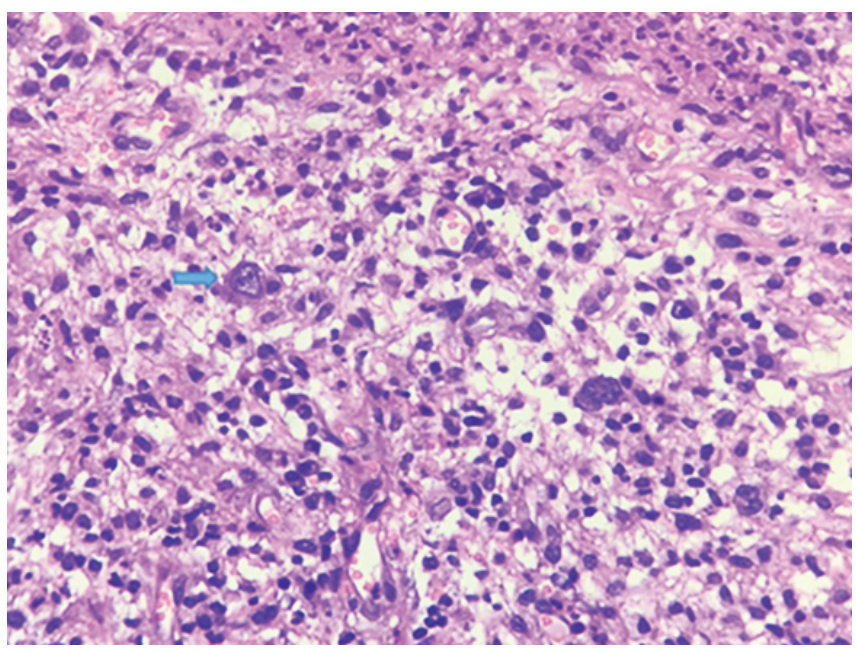

Figure 2 Dense mixed inflammatory infiltrate composed predominantly of lymphocytes, histiocytes and neutrophils admixed with large atypical, mononuclear to some binuclear cells, with prominent nucleoli, reminiscent of Hodgkin/Reed-Sternberg (HRS) cells seen in cHL (marked with arrow).

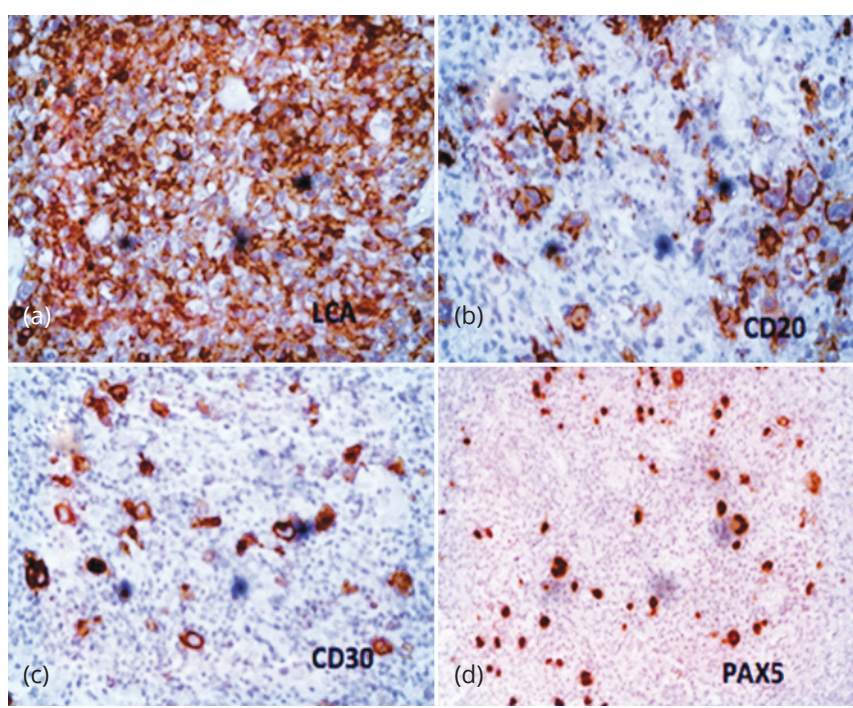

Figure 3 Large pleomorphic cells were immunonegative for LCA (Leucocyte common antigen) (Figure a) and were immunopositive for CD20, CD30 and PAX5 (Figure 3b, c \& d).

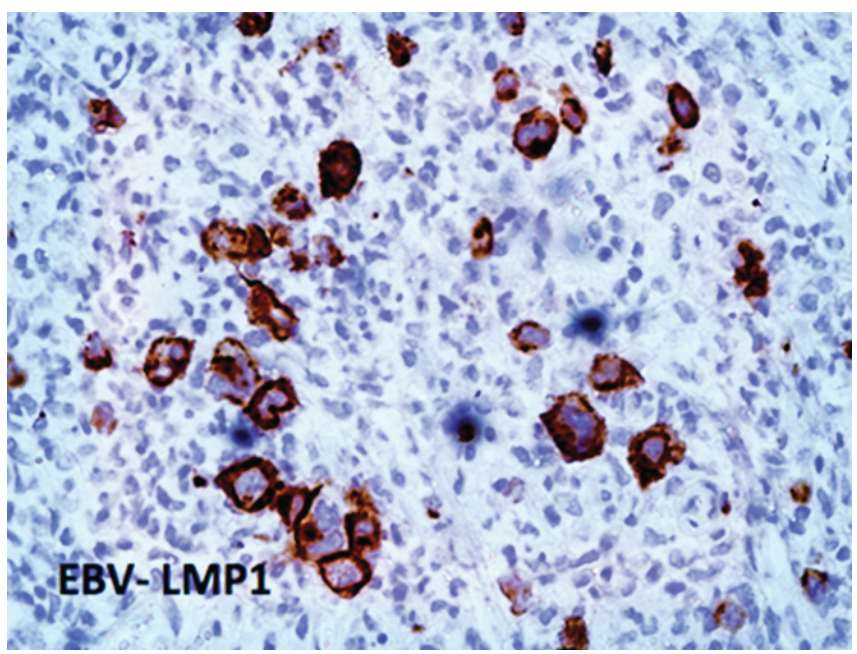

Figure 4 Immunohistochemistry for EBV-LMP1 (Epstein-Barr virus-encoded latent membrane protein 1). 


\section{Discussion}

In 2010, Dojcinov et al. [2] first described EBVMUC in a series of 26 patients associated with immunosuppression, 17 patients had age related immunosenescence, while nine cases had been treated with one or more of the immunosuppressive drugs, these included azathioprine, methotrexate or cyclosporin-A. Subsequently it was also observed in seven patients in a cohort of 70 transplant recipients with EBV post-transplant lymphoproliferative disorder (PTLD) [3]. In 2015, Bunn et al. [5] described two cases of AIDS related EBVMUC.

Epstein-Barr virus (EBV) has been associated with B-cell lymphoproliferative disorders in patients with various types of immunosuppression. After primary infection occurring at an early age EBV persists in B-cells of most adults. In immunocompetent states, the propensity of EBV to induce $B$-cell proliferation is counterbalanced by immunologic mechanisms that maintain the overall number of EBV infected B-cells in the body [6]. Immunosuppression allows EBV infected B-cells to proliferate, resulting in immunodeficiency-associated lymphoproliferative disorders. EBVMUC are lymphoproliferative lesions that present as localized cutaneous and mucosal ulcers. Although the lesions exhibit aggressive-appearing morphologic features, however, the clinical course is benign $[2,3]$. On immune morphologic grounds the cases could be classified as classical Hodgkin lymphoma. The main differentiating [3] feature between EBV MCU and classical Hodgkin lymphoma is its presentation as an isolated ulcerating lesion without a tumour mass and no detectable lesions at other sites by detailed imaging studies and bone marrow examination. The lack of detectable EBV DNA in peripheral blood is an additional distinguishing feature of EBV MCU as compared to elevated EBV DNA in other categories of PTLD [3]. Therefore, this appears to be a localized mucosal EBV- driven lymphoproliferation rather than a generalized, systemic infection as in PTLD and other immunodeficiency states. However, in this patient serum EBV DNA was not done at the time of diagnosis or later.

The limited number of case studies in literature show that most of the cases of EBV MCU respond to reduction/ discontinuation of the immunosuppressive therapy with almost complete resolution [2, 3]. After the diagnosis, the dose of azathioprine was reduced and we await follow-up after six months.

\section{Conclusion}

EBV-associated mucocutaneous ulcer is a new rare entity associated with common pathogenetic mechanism of immunosuppression, characterized by a circumscribed, isolated, shallow cutaneous or mucosal ulceration and has an indolent behaviour and a self-limited course. The morphologic and immunophenotypic features resemble $\mathrm{cHL}$, and lack of knowledge of this as an entity distinct from $\mathrm{cHL}$ can lead to an erroneous diagnosis. Therefore, correct recognition of the entity is essential to avoid overtreatment as lymphoma.

\section{Conflicts of interest}

Authors declare no conflict of interest.

\section{References}

[1] Swerdlow SH, Campo E, Pileri SA, Harris NL, Stein H, et al. The 2016 revision of the World Health Organization classification of lymphoid neoplasms. Blood. 2016; 127(20):2375-2390.

[2] Dojcinov SD, Venkataraman G, Raffeld M, Pittaluga S, Jaffe ES. EBV positive mucocutaneous ulcer-a study of 26 cases associated with various sources of immunosuppression. Am J Surg Pathol. 2010; 34(3):405-417.

[3] Hart M, Thakral B, Yohe S, Balfour HH, Singh C, et al. EBV-positive mucocutaneous ulcer in organ transplant recipients: A localized indolent posttransplant lymphoproliferative disorder. Am J Surg Pathol. 2014; 38(11):1522-1152.

[4] Weinshel EL, Peterson BA. Hodgkin's Disease. CA Cancer J Clin. 1993; 43(6):327-346.

[5] Bunn B, Heerden WV. EBV-positive mucocutaneous ulcer of the oral cavity associated with HIVIAIDS. Oral Surg Oral Med Oral Pathol Oral Radiol. 2015; 120(6):725-732.

[6] Leeuwen MT, Grulich AE, Webster AC, McCredie MR, Stewart JH, et al. Immunosuppression and other risk factors for early and late non-Hodgkin lymphoma after kidney transplantation. Blood. 2009; 114(3):630-637. 